\title{
Kinetic and Thermodynamic Studies of Charge-Transfer Complex Formation between Imipramine and 2,3-Dichloro-5,6-dicyano-1,4-benzoquinone (DDQ) in Acetonitrile and Dichloromethane Solutions
}

\author{
Masoumeh Hasani ${ }^{\mathrm{a}, *}$ and Masoud Shariati-Rad ${ }^{\mathrm{b}}$ \\ ${ }^{a}$ Faculty of Chemistry, Bu-Ali Sina University, Hamedan, 65174, Iran. \\ ${ }^{b}$ Faculty of Chemistry, Razi University, Kermanshah, Iran. \\ Received 6 January 2015, revised 17 July 2015, accepted 21 July 2015.
}

\begin{abstract}
Spectro-kinetic studies revealed the formation of charge-transfer (CT) complex of imipramine as an electron donor with $\pi$ acceptor 2,3-dichloro-5,6-dicyano-1,4-benzoquinone (DDQ) in acetonitrile and dichloromethane solutions. The resulted CT complexes exhibit spectra that were remarkably different from those of the donor and acceptor. The stoichiometry of the resulting complex was found to be 1:1 by the method of Job's continuous variation. The formation constants and thermodynamic parameters of the resulting electron-donor-acceptor (DA) complexes were determined by Benesi-Hildebrand and van't Hoff equations, respectively. The time-dependent spectra recorded after mixing donor and acceptor has been related to an immediate formation of DA complex, which is followed by two relatively slow consecutive reactions. The pseudo-first-order rate constants for the formation of the ionic intermediate and the final product have been evaluated at various temperatures by computer fitting of the absorbance-time data to appropriate equations. The activation parameters, i.e. activation energy, enthalpy, and entropy of activation were computed from temperature dependence of the rate constants. The observed results afford evidence concerning the critical role of solvent polarity on the kinetics and stability of the resulting charge transfer complexes. The ionization potential of the donor in the two solvents was estimated and compared with the theoretical values.
\end{abstract}

KEYWORDS

Charge-transfer complex, imipramine, DDQ, ionization potential, kinetic, thermodynamic.

\section{Introduction}

Charge-transfer phenomena was introduced by Mulliken ${ }^{1,2}$ and widely discussed by Foster to define a new type of adducts. Molecular interactions between electron donors and acceptors are generally associated with the formation of intensely coloured charge-transfer complexes either in the visible or UV region., ${ }^{3,4}$ These complexes are commonly found as intermediates in a wide variety of reactions involving electron-rich species or donors (D), such as nucleophiles and bases, and electrondeficient acceptors (A).

Weiss proposed ${ }^{4}$ that all molecular complexes have an essentially ionic structure $\mathrm{D}^{+} \mathrm{A}^{-}$, and pointed out that a low ionization potential for the donor $\mathrm{D}$, and a high electron affinity for the acceptor A, should then favour a stable complex. He attributed the colour of molecular complexes to intense charge-resonance spectra arising within the ions in the complex. Indeed, the ubiquitous CT absorptions are diagnostic of a very wide spectrum of intermolecular electron donor-acceptor (DA) interactions arising in extremely stable, isolable 1:1 complexes on one hand, ${ }^{5}$ to highly transient complexes (with collisional lifetimes) at the other extreme. $^{6}$

Charge-transfer interactions are very important in the field of drug-receptor binding mechanisms, ${ }^{7}$ in solar energy storage ${ }^{8,9}$ and in surface chemistry ${ }^{10}$ as well as in many biological fields. ${ }^{11}$ Charge-transfer complexes are known to take part in many chemical reactions like, addition, substitution and condensa* To whom correspondence should be addressed. E-mail: hasani@basu.ac.ir tion. ${ }^{12,13}$ Recently charge transfer complexes have been gaining importance as potential high efficiency non-linear optical materials. ${ }^{14}$ These complexes have been studied in organic conductors and photoconductors. ${ }^{15,16}$

Imipramine is commonly used to treat the depressive disorders owing to its efficiency in elevating the mood of patients by interfering to the re-uptake of norepinephrine or serotonin..$^{17}$ The study of CT interaction of imipramine with different acceptors can help to elucidate many chemical and biological phenomena that imipramine participates in. Review of the literature reveals that some spectroscopic and kinetic studies on the interaction of different drugs with DDQ has been reported before, ${ }^{8-21}$ but to the best of our knowledge there is not a systematic study on the activation parameters, stability and stoichiometry of the imipramine complex with DDQ.

The objective of the present article is to study the kinetic, thermodynamic, and mechanism of the interaction of the electron acceptor DDQ and imipramine in the solvents with different polarities.

\section{Experimental}

2,3-dichloro-5,6-dicyano-1,4-benzoquinone (DDQ) was commercially available from Merck and used without any further purification. Spectroscopic grade dichloromethane (DCM) and acetonitrile (AN) were purchased from Merck and used as received. Imipramine (antidepressant) was obtained as a gift sample from locally available pharmaceutical company. The 
<smiles>CN(C)CCCN1c2ccccc2CCc2ccccc21</smiles>

Imipramine<smiles>N#CC1=C(C#N)C(=O)C(Cl)=C(Cl)C1=O</smiles>

DDQ
Figure 1 The structure of DDQ and imipramine.

structures of imipramine and DDQ are shown in Fig. 1. The purity of the drug was verified by the measurement of its melting point $\left(174.5^{\circ} \mathrm{C}\right) .^{22}$ Stock solutions of DDQ and imipramine freshly prepared before each series of measurements by dissolving precisely weighed amounts of the component in the appropriate volume of solvent. Diluting appropriate volumes of stock donor and DDQ solutions with pure solvent were used to make working solutions for spectroscopic measurements.

The electronic absorption spectra of the studied CT complex solutions were recorded using a Perkin-Elmer Lambda 45 spectrophotometer with a pair of matched quartz cells of $1.0 \mathrm{~cm}$ path length. Rate measurements were performed on a Shimadzu UV-265 spectrophotometer with a CPS-260 thermostat for controlling temperature with precision of $\pm 0.1^{\circ} \mathrm{C}$. The molecular orbital package, MOPAC 2000 version 6.0 (PM3 method) was used for the theoretical calculation of the ionization potential of the donor. ${ }^{23}$

\section{Results and Discussion}

\subsection{Electronic Spectral Studies}

Imipramine as an electron donor interacts with DDQ as an electron acceptor (A), to form a charge-transfer complex. Fig. 2 shows the UV-vis absorption spectra of mixtures of DDQ in
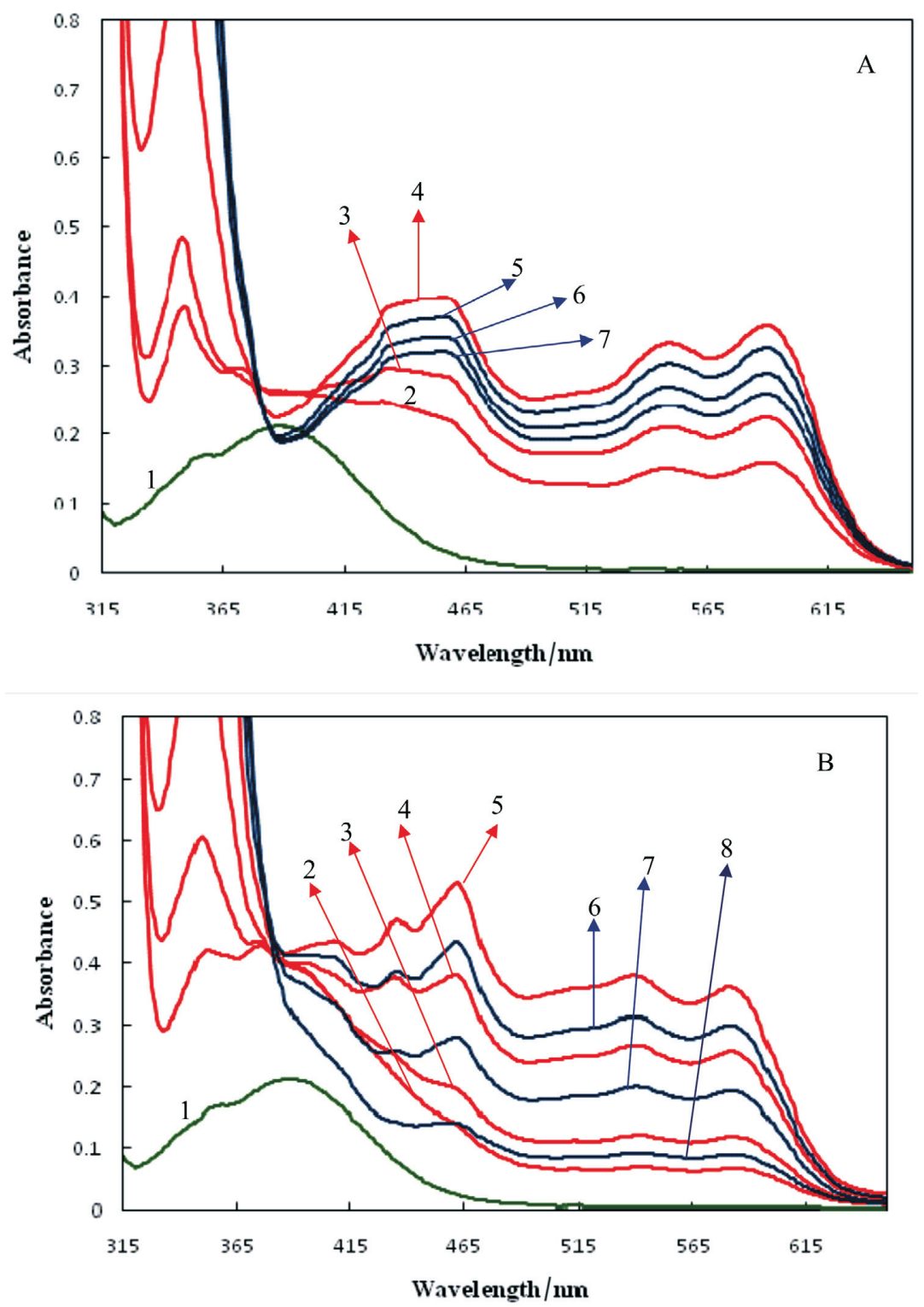

Figure 2 (A) Electronic absortion spectra in the reaction of DDQ $\left(2.5 \times 10^{-4} \mathrm{M}\right)$ and imipramine $\left(1.46 \times 10^{-2} \mathrm{M}\right)$ in acetonitrile at $25^{\circ} \mathrm{C}$. (1) DDQ alone, (2) immediately after mixing and at time intervals (3) $1 \mathrm{~min},(4) 10 \mathrm{~min}$, (5) $30 \mathrm{~min}$, (6) $40 \mathrm{~min}$, (7) $48 \mathrm{~min}$. (B) in dichloromethane (1) DDQ alone, (2) immediately after mixing and at time intervals (3) $1 \mathrm{~min}$, (4) $5 \mathrm{~min}$, (5) $10 \mathrm{~min}$, (6) $16 \mathrm{~min}$, (7) $25 \mathrm{~min}$, (8) $40 \mathrm{~min}$. 
acetonitrile (AN) and dichloromethane (DCM) solutions with an excess amount of imipramine (i.e. [imipramine]/[DDQ] $=58$ to attain pseudo-first-order conditions) as a function of time at $25^{\circ} \mathrm{C}$. While none of the reactants show any considerable absorption in the $450-600 \mathrm{~nm}$ region, addition of imipramine to DDQ results in strong absorptions in this spectral region, presumably due to the occurrence of charge transfer during the formation of corresponding molecular complexes. The resulting spectra, after mixing of DDQ with imipramine, are characterized by maximum absorptions at the wavelengths $588,547,465$, and $350 \mathrm{~nm}$. These spectral characteristics are highly correlated to the spectra reported for $\mathrm{DDQ}^{\cdot-}$ radical anion ${ }^{24-26}$ due to the inner-sphere character of the formed charge-transfer complex. Examination of the literature ${ }^{24,27-30}$ shows that in many donoracceptor systems with DDQ as an acceptor, the formation of DDQ anion radical is often noticed which are only obtained through the formation of inner complexes from the initially formed outer complexes. Mulliken ${ }^{31}$ pointed out that a donoracceptor pair would either form the associative outer complex or the dissociative inner complex depending on the distance of approach between the donor and acceptor and the relative magnitude of the no-bond and dative wave functions. It was also suggested that the formation of the inner complex from the outer complex should be strongly dependent on environmental conditions. Early evidence for the transformation of outer complexes to the inner ones in charge-transfer complex formation is the electrical conductance, which was explained ${ }^{32,33}$ on the basis of the equilibrium between outer and inner complexes.

Obviously, the spectra recorded for the complexes between DDQ and imipramine are time dependent. With elapse of time, the mentioned spectral characteristics became more evident and a new peak located at 340-360 nm appears. It should be mentioned that, the spectral changes in the region of $340-360 \mathrm{~nm}$ continue to increase very slowly till the end of the time of monitoring accompanied by decrease in absorbance at 450-600 nm. The observed gradual decrease in the intensity of the chargetransfer bands in the 450-600 $\mathrm{nm}$ spectral regions could be due to the consumption of the ionic intermediates through an irreversible chemical reaction, ${ }^{34}$ while the continuous increase of the $340-360 \mathrm{~nm}$ bands with elapse of time is indicative of the formation of the final reaction product.

Mulliken described the new low energy absorptions observed in solutions containing both a donor and an acceptor as chargetransfer transitions..$^{35}$ This low energy charge-transfer transition involves promotion of an electron residing in the highest occupied molecular orbital (HOMO) of the donor to the lowest unoccupied molecular orbital (LUMO) of the acceptor as shown for $\mathrm{h} v_{\mathrm{CT}}{ }^{35}$

The experimental observations seem to be in accord with the mechanism illustrated below, as proposed before..$^{34,36-38}$

$$
\begin{array}{ll}
\mathrm{D}+\mathrm{A} \stackrel{\mathrm{K}_{1}}{\longrightarrow} \text { D.A } & \text { very fast } \\
\text { D.A } \stackrel{\mathrm{k}_{1}}{\longrightarrow} \mathrm{D}^{\bullet+}+\mathrm{A}^{\cdot-} & \text { slow } \\
\mathrm{D}^{\bullet+}+\mathrm{A}^{\cdot-} \stackrel{\mathrm{k}_{2}}{\longrightarrow} \mathrm{P} & \text { slow }
\end{array}
$$

According to the proposed mechanism, the first step involves a fast equilibrium for the formation of a D.A complex between imipramine (D) and DDQ (A), which is followed by two relatively slow consecutive reactions.

The observed enhanced absorption band intensities, immediately after mixing $\mathrm{D}$ and $\mathrm{A}$, supports the fact that the chargetransfer complex formed is of the dative-type structure which consequently converts to an ionic intermediate possessing the spectral characteristics of DDQ radical ion.

\subsection{Kinetic Study}

In order to investigate the kinetics of production and consumption of DDQ ${ }^{--}$radical anions (i.e. $\mathrm{k}_{1}$ and $\mathrm{k}_{2}$ ), the absorbance at $590 \mathrm{~nm}$ was monitored as a function of time in solutions containing reactants at an imipramine-to-DDQ mole ratio of 20 at various temperatures. The resulting absorbance-time plots for the imipramine-DDQ system in both DCM and AN solutions at different temperatures are shown in Fig. 3.

For the pair of consecutive reactions given in Eqs. (2) and (3), the concentrations of species involved as a function of time, under the pseudo-first-order conditions, are given by Eqs. (4) $-(6):{ }^{39}$

$$
\begin{aligned}
& {[\text { D. } A]=[D \cdot A]_{0} \mathrm{e}^{-\mathrm{k}_{1} \mathrm{t}}} \\
& {\left[\mathrm{D}^{\bullet+}\right]=\left[\mathrm{A}^{\bullet-}\right]=\frac{[\mathrm{D} \cdot \mathrm{A}]_{0} \mathrm{k}_{1}}{\mathrm{k}_{2}-\mathrm{k}_{1}}\left(\mathrm{e}^{-\mathrm{k}_{1} \mathrm{t}}-\mathrm{e}^{-\mathrm{k}_{2} \mathrm{t}}\right)} \\
& {[\mathrm{P}]=[D \cdot A]_{0}+\frac{[\mathrm{D} \cdot \mathrm{A}]_{0}}{\mathrm{k}_{1}-\mathrm{k}_{2}}\left(\mathrm{k}_{2} \mathrm{e}^{-\mathrm{k}_{1} \mathrm{t}}-\mathrm{k}_{1} \mathrm{e}^{-\mathrm{k}_{2} \mathrm{t}}\right)}
\end{aligned}
$$

where $[\mathrm{D} . \mathrm{A}]_{0},\left[\mathrm{D}^{\cdot+}\right],\left[\mathrm{A}^{\cdot-}\right]$, and $[\mathrm{P}]$ are the initial concentration of the D.A complex, the intermediate cation and anion radicals concentrations, and the final product concentration, respectively. $k_{1}$ and $k_{2}$ are the rate constants of the reactions (2) and (3), respectively.

The absorbance of the reaction solution at time $t$ is given by Eq. (7): ${ }^{39}$

$$
\mathrm{A}_{\mathrm{t}}=\varepsilon_{\mathrm{D} . \mathrm{A}}[\mathrm{D} . \mathrm{A}]+\varepsilon_{\mathrm{A}^{\bullet-}}\left[\mathrm{A}^{\bullet-}\right]+\varepsilon_{\mathrm{p}}[\mathrm{P}]
$$

where $\varepsilon_{\mathrm{D}, \mathrm{A}}, \varepsilon_{\mathrm{A} \cdot--}$, and $\varepsilon_{\mathrm{P}}$ are the molar absorptivities of species D.A, $\mathrm{A}^{\cdot-}$ and product, respectively. Based on our observation, the
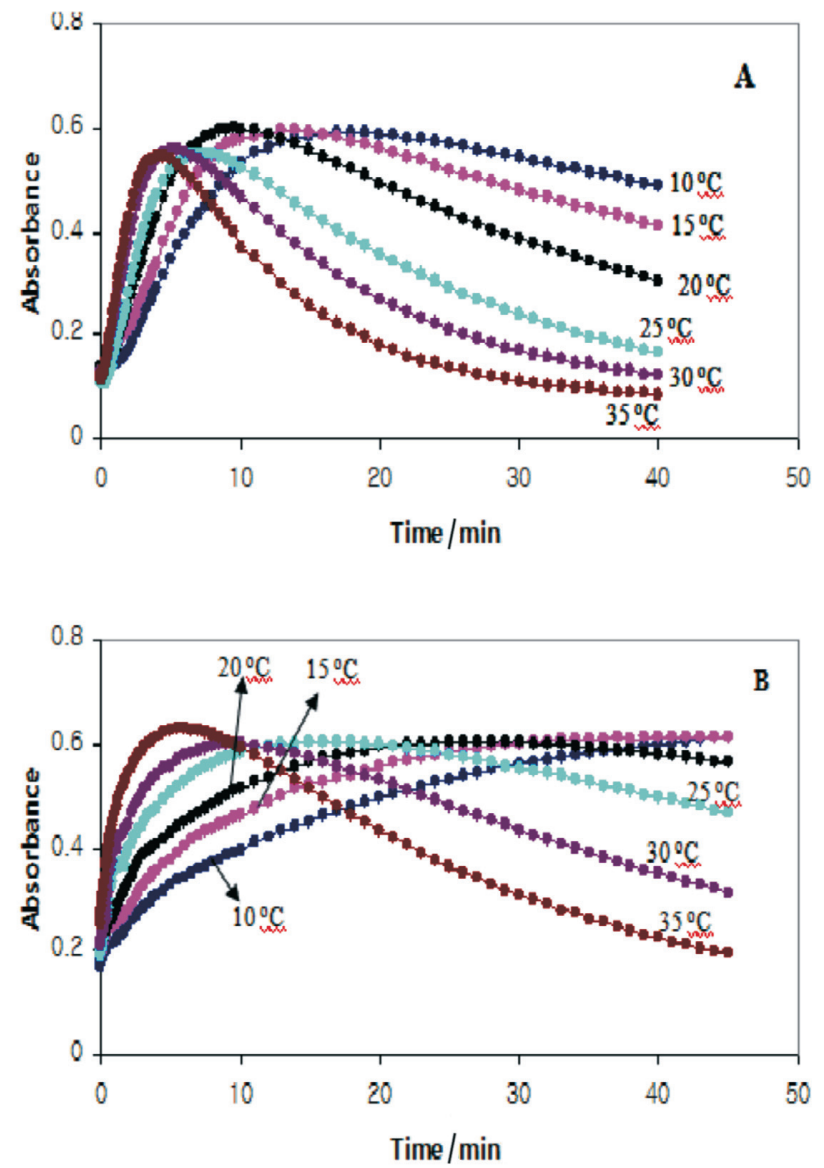

Figure 3 Plots of absorbance versus time for a mixture of DDQ $\left(2.4 \times 10^{-4}\right.$ $\mathrm{M})$ and imipramine $\left(4.8 \times 10^{-3} \mathrm{M}\right)$ in $(\mathbf{A})$ acetonitrile and $(\mathbf{B})$ dichloromethane solutions at different temperatures. 
monitored absorbance at $590 \mathrm{~nm}$ decreases with elapse of time and finally reaches very near to zero. So, the product $P$ was set as nonabsorbing species $\left(\varepsilon_{\mathrm{P}}=0\right)$.

The substitution of Eqs (4) and (5) in Eq. (7) and rearrangements results in:

$$
A_{t}=[D \cdot A]_{0}\left(\varepsilon_{D \cdot A} e^{-k_{1} t}+\left(k_{1} e_{A^{\cdot-}} \times \frac{e^{-k_{2} t}-e^{-k_{1} t}}{k_{1}-k_{2}}\right)\right)
$$

The pseudo-first-order rate constants $k_{1}$ and $k_{2}$ at various temperatures were then evaluated by fitting the corresponding experimental absorbance-time data to Eq. (8) using a non-linear least squares curve-fitting program KINFIT. $^{40}$ The program is based on the iterative adjustment of calculated to the observed absorbance values by using either the Wentworth matrix technique $^{41}$ or the Powell procedure. ${ }^{42}$ The adjustable parameters are $\mathrm{k}_{1}, \mathrm{k}_{2}, \varepsilon_{\mathrm{D} . \mathrm{A}^{\prime}}$ and $\varepsilon_{\mathrm{A}} \ldots$. The output of the KINFIT program comprises the refined parameters, the sum of squares and the standard deviation of the data. All the kinetic and thermodynamic data have been repeated three times. A sample fit of the absorbancetime data is shown in Fig. 4. A fair agreement between the observed and calculated absorbances further supports the

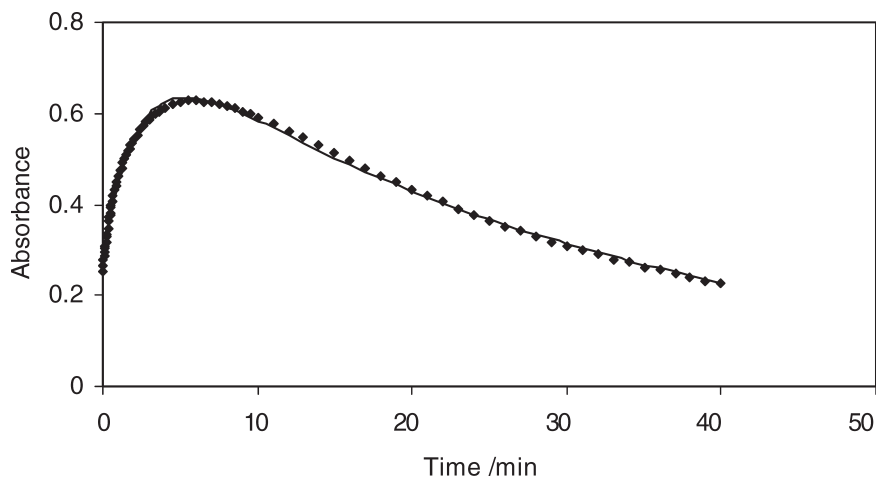

Figure 4 Measured and calculated absorbance as a function of time for $2.4 \times 10^{-4} \mathrm{M}$ of DDQ and $4.8 \times 10^{-3} \mathrm{M}$ imipramine in dichloromethane at $20{ }^{\circ} \mathrm{C}$. The nonlinear least-square fit according to Eq. (8) is shown as a solid line. occurrence of reactions between the imipramine and DDQ via the two-step mechanism suggested.

All the values obtained for $\mathrm{k}_{1}$ and $\mathrm{k}_{2}$ at various temperatures are summarized in Table 1 . The activation parameter, $\mathrm{E}_{\mathrm{a}}$, and transition state parameters, $\Delta \mathrm{H}^{\#}$ and $\Delta \mathrm{S}^{\#}$, were then calculated by using the corresponding Arrhenius plots and the Eyring transition-state theory, ${ }_{1}^{43}$ respectively; the results are given in Table 2.

The data given in Table 1 indicate that, in all cases studied, the pseudo-first-order rate constants increase with increasing temperature. It is also obvious that, the $\mathrm{k}_{1}$ values in AN solution are larger than those in DCM solution, while, an opposite solvent effect is observed on the rate of the second steps of the reactions, $k_{2}$, the $k_{2}$ values in AN are smaller than those observed in DCM solution. It should be noted that the observed solvent effect on the $k_{1}$ and $k_{2}$ values is in support of the proposed two-step mechanism. The resulting DDQ ${ }^{--}$radical ions from the first step are expected to become more stabilized in AN as a solvent of higher solvating ability and dielectric constant than $\mathrm{DCM} .{ }^{44}$ Consequently, the rate of production of $\left[\mathrm{A}^{--}\right]$is expected to increase in AN solution, while its consumption to the final product should be decreased in this solvent. This behaviour has been reported before. ${ }^{28}$

The $\mathrm{E}_{\mathrm{a}}$ and $\Delta \mathrm{H}^{\#}$ values (Table 2 ) are in complete accordance with the values of rate constants. The larger are these parameters, the smaller are the rate constants. From Table 2 it is obvious that, although the rate of the first step of the reaction is higher than that of the second step in DCM, it is associated with higher activation energy as it compared with the value of activation energy for the second step. This may be explained in terms of the compensatory effect of highly negative $\Delta \mathrm{S}^{\#}{ }_{2}$ values on the reaction rate. Thus, the less negative $\Delta S^{\#}{ }_{1}$ for the production of $\mathrm{DDQ}^{--}$radical anions may result in the higher rate constants, as it compared with the more negative $\Delta S^{\#}{ }_{2}$ for its consumption, although from the observed trend in $\Delta \mathrm{H}^{\#}$ values an opposite behavior is expected.

For supporting of the formation of ionic species in solution, the conductivity of the solution was measured before and after addition of imipramine to DDQ. The increase in the conductivity

Table 1 Rate constants for charge-transfer complex formation between DDQ and imipramine at different temperatures $(n=3)$.

\begin{tabular}{|c|c|c|c|}
\hline Solvent & Temperature $/{ }^{\circ} \mathrm{C}$ & $\mathrm{k}_{1} / \min ^{-1}$ & $\mathrm{k}_{2} / \mathrm{min}^{-1}$ \\
\hline \multirow[t]{6}{*}{$\mathrm{AN}$} & 10 & $6.40 \times 10^{-2} \pm 1.00 \times 10^{-3}$ & Too slow \\
\hline & 15 & $1.03 \times 10^{-1} \pm 1.00 \times 10^{-3}$ & Too slow \\
\hline & 20 & $1.54 \times 10^{-1} \pm 5.00 \times 10^{-3}$ & $1.90 \times 10^{-3} \pm 4.01 \times 10^{-4}$ \\
\hline & 25 & $2.41 \times 10^{-1} \pm 9.00 \times 10^{-3}$ & $7.80 \times 10^{-3} \pm 4.01 \times 10^{-4}$ \\
\hline & 30 & $3.34 \times 10^{-1} \pm 1.00 \times 10^{-2}$ & $1.82 \times 10^{-2} \pm 4.01 \times 10^{-4}$ \\
\hline & 35 & $4.51 \times 10^{-1} \pm 8.00 \times 10^{-3}$ & $3.16 \times 10^{-2} \pm 3.00 \times 10^{-4}$ \\
\hline \multirow[t]{6}{*}{ DCM } & 10 & $4.50 \times 10^{-2} \pm 1.20 \times 10^{-2}$ & Too slow \\
\hline & 15 & $9.60 \times 10^{-2} \pm 8.02 \times 10^{-3}$ & $3.15 \times 10^{-2} \pm 3.10 \times 10^{-3}$ \\
\hline & 20 & $1.48 \times 10^{-1} \pm 8.10 \times 10^{-3}$ & $3.61 \times 10^{-2} \pm 2.10 \times 10^{-3}$ \\
\hline & 25 & $2.16 \times 10^{-1} \pm 9.02 \times 10^{-3}$ & $4.93 \times 10^{-2} \pm 1.01 \times 10^{-3}$ \\
\hline & 30 & $3.37 \times 10^{-1} \pm 1.01 \times 10^{-2}$ & $5.70 \times 10^{-2} \pm 1.00 \times 10^{-3}$ \\
\hline & 35 & $4.47 \times 10^{-1} \pm 1.70 \times 10^{-2}$ & $7.44 \times 10^{-2} \pm 2.20 \times 10^{-3}$ \\
\hline
\end{tabular}

Table 2 Transition state parameters and activation parameters for charge-transfer complex formation between DDQ and imipramine $(n=3)$.

\begin{tabular}{lccccc}
\hline Solvent & $\begin{array}{c}\Delta \mathrm{H}^{\#}{ }_{1} \\
/ \mathrm{kJ} \mathrm{mol}^{-1}\end{array}$ & $\begin{array}{c}\Delta \mathrm{H}^{\#}{ }_{2} \\
/ \mathrm{kJ} \mathrm{mol}^{-1}\end{array}$ & $\begin{array}{c}\Delta \mathrm{S}^{\#}{ }_{1} \\
/ \mathrm{J} \mathrm{mol}^{-1} \mathrm{~K}^{-1}\end{array}$ & $\begin{array}{c}\Delta \mathrm{S}^{\#}{ }^{2} \\
/ \mathrm{Jmol}^{-1} \mathrm{~K}^{-1}\end{array}$ & $\begin{array}{c}\mathrm{Ea}_{1} \\
/ \mathrm{kJ} \mathrm{mol}^{-1}\end{array}$ \\
\hline $\mathrm{AN}$ & $54.3 \pm 1.1$ & $140.1 \pm 13.7$ & $-109.1 \pm 3.6$ & $-162.3 \pm 33.8$ & $55.5 \pm 8.1$ \\
$\mathrm{ECM}$ & $62.4 \pm 3.0$ & $29.6 \pm 1.6$ & $-82.3 \pm 10.2$ & $-190.7 \pm 5.5$ & $64.9 \pm 17.0$ \\
\hline
\end{tabular}


of DDQ solution after addition of imipramine indicates the formation of ionic species in the solution. It was also found that the formation of ionic species is dependent to the solvent polarity. A higher value of conductivity of CT complex in AN with a higher dielectric constant was obtained compared with that in DCM with lower polarity.

\subsection{Equilibrium Study}

Job's method of continuous variation, ${ }^{45}$ which provided symmetrical curves with maxima at a mole fraction of 0.5 , in both the solvents, confirms a 1:1 stoichiometric ratio (Fig. 5). The equilibrium constants of the formation of imipramine-DDQ charge-transfer complexes in the two solvents were determined employing the absorbance at $590 \mathrm{~nm}$. As seen in Fig. 6 during the first few seconds after the addition of imipramine to DDQ

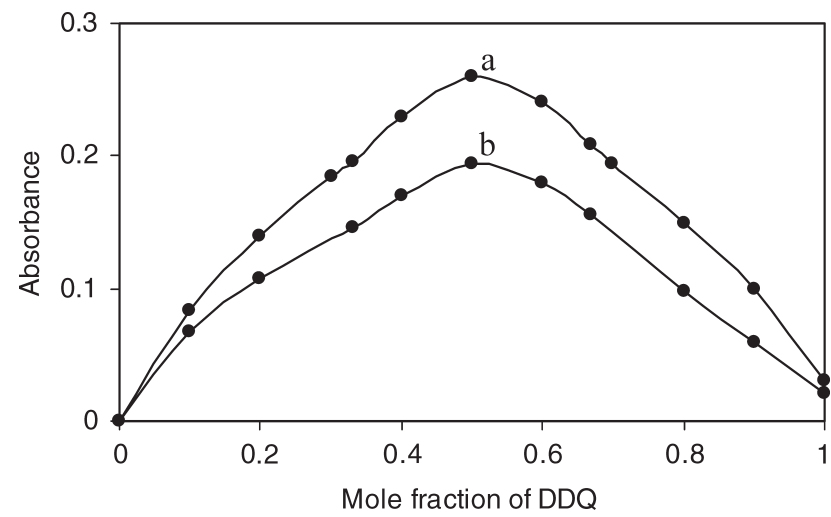

Figure 5 Continuous variation plots for (a) DDQ-imipramine system in acetonitrile and (b) in dichloromethane at $25^{\circ} \mathrm{C} . \lambda=590 \mathrm{~nm}(n=2)$. solution, a rather sharp step-functional increase in the absorbance at $590 \mathrm{~nm}$ was observed. This initial change in the absorbance $(\Delta \mathrm{A})$ upon the mixing of imipramine and DDQ was found to increase along with a rise in the concentration of imipramine.

$\Delta \mathrm{A}$ values at $590 \mathrm{~nm}$ were measured using constant acceptor concentration (in a given solvent) and varying concentration of a donor but always $[\mathrm{D}]>>[\mathrm{A}]$. The formation constants $\left(K_{\mathrm{CT}}\right)$ and molar extinction coefficients $\left(\varepsilon_{\mathrm{CT}}\right)$ of the charge-transfer complexes were determined spectrophotometrically in different temperatures using the Benesi-Hildebrand equation: ${ }^{46}$

$$
\frac{[\mathrm{A}]_{0}}{\Delta \mathrm{A}}=\frac{1}{\mathrm{~K}_{\mathrm{CT}} \varepsilon_{\mathrm{CT}}} \frac{1}{[\mathrm{D}]}+\frac{1}{\varepsilon_{\mathrm{CT}}}
$$

where $[D]$ and $[A]_{0}$ are the initial molar concentration of the donor and acceptor, respectively. The values of $\mathrm{K}_{\mathrm{CT}}$ and $\varepsilon_{\mathrm{CT}}$ are determined from the slope and intercept of the linear plot of $[\mathrm{A}]_{0} / \Delta \mathrm{A}$ against $1 /[\mathrm{D}]$. A representative plot is shown in Fig. 7. The linearity of the plots of the Benesi-Hildebrand equation supports the stoichiometry of 1:1 of the charge-transfer complexes elucidated by Job's method.

The results given in Table 3 revealed that, the stability of the resulting charger-transfer complexes increases with increasing polarity of the solvent from DCM to AN. A similar solvent effect on the stability and charge transition energies of different charge-transfer complexes has been reported in the literature. ${ }^{47-52}$. It has been suggested that the observed trend in the stability of the charge-transfer complexes could be due to the high stabilization of the excited states in which the charge is probably more separated than in the ground states.

The thermodynamic parameters, enthalpy change $\left(\Delta \mathrm{H}^{0}\right)$ and

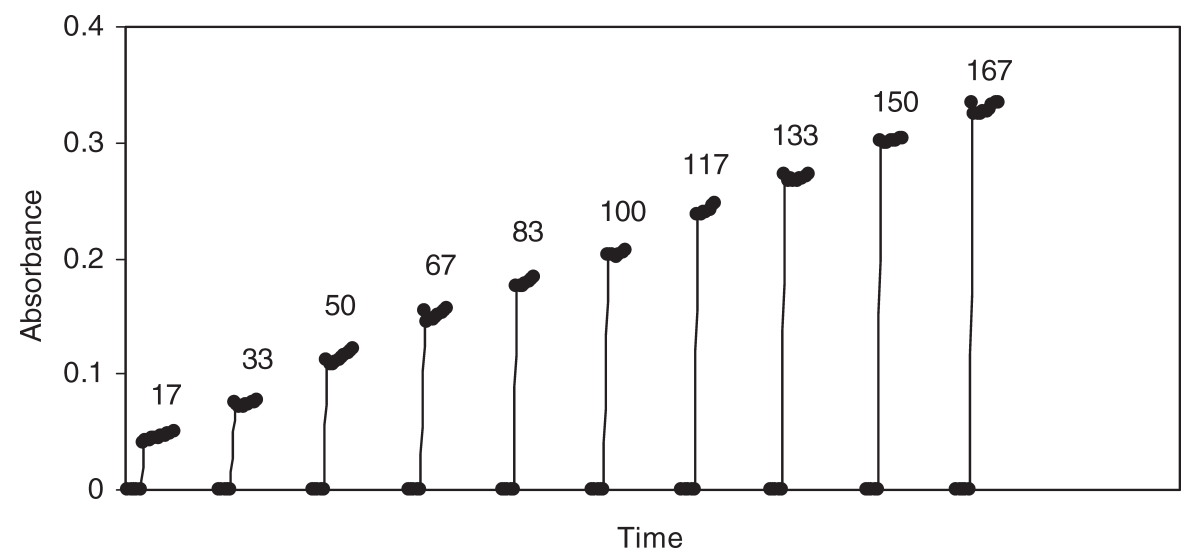

Figure 6 Absorbance-time changes upon addition of imipramine to the solution of DDQ $\left(2.4 \times 10^{-4} \mathrm{M}\right)$ in dichloromethane solution at $10{ }^{\circ} \mathrm{C}$. The values on the curves denote the ratio of the donor to the acceptor $(n=2)$.

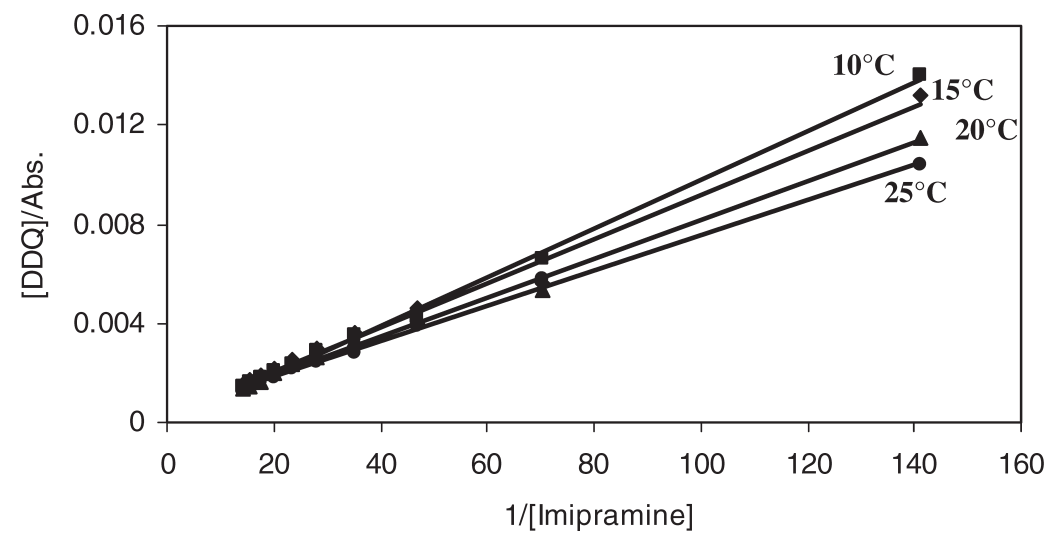

Figure 7 Benesi-Hildebrand plots for DDQ-imipramine system in dichloromethane at different temperatures $(n=3)$. 
Table 3 Charge-transfer complex formation constant and thermodynamic parameters evaluated from Benesi-Hildebrand equation at different temperatures $(n=3)$.

\begin{tabular}{lcrccc}
\hline Solvent & $\begin{array}{c}\text { Temperature } \\
\text { / } \mathrm{C}\end{array}$ & $\mathrm{K}_{\mathrm{CT}}$ & $\varepsilon_{\mathrm{CT}}$ & $\begin{array}{c}\Delta \mathrm{H}^{\circ} \\
/ \mathrm{kJ} \mathrm{mol}^{-1}\end{array}$ & $\begin{array}{c}\Delta \mathrm{S}^{\circ} \\
/ \mathrm{J} \mathrm{mol}^{-1} \mathrm{~K}^{-1}\end{array}$ \\
\hline AN & 10 & $12.7 \pm 1.5$ & $4452 \pm 995$ & $-54.2 \pm 6.5$ & $-170.4 \pm 37.6$ \\
& 20 & $5.6 \pm 1.2$ & & & \\
& 25 & $4.0 \pm 0.6$ & & & $-108.1 \pm 24.9$ \\
DCM & 35 & $1.9 \pm 0.8$ & $3215 \pm 895$ & $-34.7 \pm 8.3$ & \\
& 10 & $5.6 \pm 0.8$ & & & \\
& 15 & $4.3 \pm 1.0$ & & & \\
& 20 & $3.5 \pm 1.4$ & & & \\
\hline
\end{tabular}

entropy change $\left(\Delta S^{0}\right)$, associated with the CT complex formation of DDQ with imipramine in the two studied solvents were determined from the computed $\mathrm{K}_{\mathrm{CT}}$ values at different temperatures. The obtained results revealed that the formed complexes are better stabilized as the temperature is lowered. The observed decrease in formation constant values with rise in temperature indicates the exothermic nature of the interaction between DDQ and imipramine molecule. The values of $\Delta \mathrm{H}^{0}$ and $\Delta \mathrm{S}^{0}$ were calculated from the slope and intercept of the linear von't Hoff plot, respectively and the obtained results are also given in Table 3. These values show that the complexation is exothermic and enthalpy driven with a negative entropic contribution. The negative enthalpies show that the complex formation is spontaneous wile negative entropy indicates a decrease in the degree of freedom of the components upon complexation.

\subsection{Ionization Potential}

Out of the many applications of charge-transfer complexes, one important application is to calculate the ionization potential of the donor. The ionization potential $\left(\mathrm{I}_{\mathrm{p}}\right)$ of the HOMO of the donor has been estimated from charge-transfer energies of its complexes with the acceptor making use of the empirical equations reported in the literature..$^{53}$

$$
\mathrm{I}_{\mathrm{p}}(\mathrm{eV})=5.76+1.52 \times 10^{-4} \bar{v}\left(\mathrm{~cm}^{-1}\right)
$$

where $\bar{v}$ is the wave number corresponding to the charge-transfer band of the charge-transfer complex which is 17301 and $16949 \mathrm{~cm}^{-1}$ for charge-transfer complex formation in DCM and $\mathrm{AN}$, respectively. It has been reported that the ionization potential of the electron donor may be correlated with the charge-transfer transition energy of the complex. ${ }^{54}$ The calculated $I_{p}$ values for molecular orbital participating in chargetransfer interaction of the drug in AN and DCM are 8.34 and $8.39 \mathrm{eV}$, respectively. Moreover, MOPAC PM3 calculation of the $\mathrm{I}_{\mathrm{p}}$ of imipramine resulted in $8.52 \mathrm{eV}$. In the present study, the theoretical (MOPAC PM3 method) and experimental ionization potential values are in relatively good agreement with each other. This fact supports the interpretation that the low energy band can be regarded as the charge-transfer band.

Further evidence for the nature of charge-transfer interaction in the present system is the calculation of the dissociation energy (W) of the charge-transfer excited state of the complex. In fact, it is the electrostatic energy of the ion pair $\left[\mathrm{D}^{\cdot+}, \mathrm{A}^{*-}\right]$. To the first approximation, the separation between $\mathrm{D}$ and $\mathrm{A}$ in D.A complex is inversely related to $\mathrm{W} .{ }^{55}$ The dissociation energies of the complex were calculated from their charge-transfer energy, $\mathrm{h} \overline{\mathrm{C}}_{\mathrm{CT}}$, the ionization potential of the donor, $I_{p}$ and electron affinity, $E_{A}$ of the acceptor using the empirical relation ${ }^{56}$ given in the following equation:

$$
h \bar{v}_{\mathrm{CT}}=\mathrm{I}_{\mathrm{p}}-\mathrm{E}_{\mathrm{A}}-\mathrm{W}
$$

The calculated values of $\mathrm{W}$ are 2.96 and $2.97 \mathrm{eV}$ in DCM and $\mathrm{AN}$, respectively. These $\mathrm{W}$ values suggest that the investigated complex is stable under the studied conditions with high resonance stabilization energy. ${ }^{56}$

\section{Conclusions}

Spectrophotometric studies revealed that the interaction of imipramine and 2,3-dichloro-5,6-dicyano-1,4-benzoquinone to be proceed through the formation of a 1:1 CT-complexes. The values of the obtained $\mathrm{K}_{\mathrm{CT}}$ can be indicative of a transition between the donor and the acceptor, such as the donor forms radical cation and the acceptor forms radical anion. The equilibrium process of the $\mathrm{CT}$ formation is followed by an irreversible kinetic reaction. The permittivity of the medium affects the equilibrium and kinetics of the DDQ-drug interaction. The system was characterized by the formation of the $\mathrm{DDQ}^{-{ }^{-}}$radical ion. The mechanism of the interaction of the drug studied may be useful in understanding the binding of drug molecule in real pharmacokinetic study.

\section{Acknowledgements}

The authors are grateful to the Bu-Ali Sina University for supporting this work.

\section{References}

1 R.S. Mulliken, Structures of complexes formed by halogen molecules with aromatic and with oxygenated solvents, J. Am. Chem. Soc., 1950, 72, 600-608.

2 R.S. Mulliken and W.B. Pearson, Molecular Complexes, Wiley Publishers, New York, 1969.

3 R. Foster, Organic Charge-transfer Complexes, vol. 51, Academic Press, London, 1969.

4 J. Weiss, The formation and structure of some organic molecular compounds, J. Chem. Soc., 1942, 245-252.

5 H.M. Powell, G. Huse and P.W. Cook, The structure of molecular compounds. Part I. The crystal structure of p-iodoaniline-s-trinitrobenxen, J. Chem. Soc., 1943, 153-157.

6 M. Tamres and R.L. Strong, Molecular Association, vol. 2, (R. Foster, ed.), Academic, London, 1979.

7 A. Korolkovas, Essentials of Medical Chemistry, 2nd edn., Wiley, New York, 1998.

8 K. Takahashi, K. Horino, T. Komura and K. Murata, Photovoltaic properties of porphyrin thin-films mixed with o-chloranil, Bull. Chem. Soc. Jpn., 1993, 66, 733-738.

9 W. Wu, J. Li, F. Guo, L. Zhang, Y. Long and J. Hua, Photovoltaic performance and long-term stability of quasi-solid-state fluoranthene dyes-sensitized solar cells, Renew. Energ., 2010, 35, 1724-1728.

10 S.M. Andrade, S.M.B. Costa and R. Pansu, Structural changes in W/O triton X-100/cyclohexane-hexanol/water microemulsions probed by a fluorescent drug piroxicam, J. Colloid Interface Sci., 2000, 226, 260-268.

11 A.M. Slifkin, Charge-Transfer Interaction of Biomolecules, Academic Press, New York, 1971.

12 F.P. Fla, J. Palou, R. Valero, C.D. Hall and P. Speers, Kinetics and mech- 
anism of the addition of triphenylphosphoniocyclopentadienide to tetrachloro-p-benzoquinone, J. Chem. Soc., Perkin Trans., 1991, 2 1925-1932.

13 K. Datta, M. Banerjee, B.K. Seal and A.K. Mukherjee, Ground state EDA complex formation between [60]fullerene and a series of polynuclear aromatic hydrocarbons, Chem. Soc. Perkin Trans., 2000, 2, 531-534.

14 S.D. Bella, I.L. Fragala, M.A. Ratner and T.J. Marks, Electron donoracceptor complexes as potential high-efficiency second-order nonlinear optical materials. A computational investigation, J. Am. Chem. Soc., 1993, 115, 682-686.

15 A. Eychmuller and A.L. Rogach, Chemistry and photophysics of thiol-stabilized II-VI semiconductor nanocrystals, Pure Appl. Chem., 2000 72, 179-188.

16 R. Dabestani, K.J. Reszka and M.E. Sigman, Surface catalyzed electron transfer from polycyclic aromatic hydrocarbons (PAH) to methyl viologen dication: evidence for ground-state charge transfer complex formation on silica gel, J. Photochem. Photobiol. A, 1998, 117, 223-233.

17 J.G. Hardman, L.E. Limbird, P.B. Molonoff, R.W.R. Ruddon and A.G. Gilman, Goodman and Gilman's, The Pharmacological Basis of Therapeutics, McGraw-Hill, New York, 1996.

18 M. Pandeeswaran and K.P. Elango, Spectroscopic and kinetic studies on the interaction of ketoconazole and povidone drugs with DDQ Spectrochim. Acta A, 2008, 69, 1082-1088.

19 G. G. Mohamed, F.A. Nour El-Dien and E.U. Farag, Spectrophotometric study of the reaction mechanism between DDQ as $\pi$-acceptor and potassium iodate and flucloxacillin and dicloxacillin drugs and their determination in pure and in dosage forms, Spectrochim. Acta $A$, 2006, 65, 11-19.

20 A. Fakhro, H.S. Bazzi, A. Mostafa and L. Shahada, Spectroscopic characterization and thermal structural analysis of the chargetransfer complexes formed in the reaction of 1-methylpiperazine with $\sigma$ - and $\pi$-acceptors, J. Mol. Struct., 2010, 973, 9-17.

$21 \mathrm{M}$. Hasani and S. Nikoee, Kinetic and thermodynamic studies on molecular interaction of antipyrine donor and 2,3-dichloro-5,6-dicyano-1,4-benzoquinone as an electron acceptor in different solvents, Int. J. Chem. Kinet., 2013, 45, 81-91.

22 M. Windholz and S. Budavari, eds, The Merck Index: An Encyclopedia of Chemicals, Drugs and Biologicals, 10th edn., Merck \& Co., Inc., Rattway, New Jersey, 1983.

23 J.J.P. Stewart, Optimization of parameters for semi-empirical methods I. Method, J. Comput. Chem., 1989, 10, 209-220.

24 K. Ganesh, C. Balraj, A. Satheshkumar and K.P. Elango, Spectroscopic investigation on the mechanism of formation of molecular complexes of albendazole and trimethoprim with 2,3-dichloro-5,6dicyano-1,4-benzoquinone, Spectrochim. Acta A, 2012, 92, 46-55.

25 S. Matsuzaki, T. Mitsuishi and K. Toyoda, Resonance Raman spectra of the TCNE radical anion dimer in ethanol, Chem. Phys. Lett., 1982, 91, 296-298.

26 J.S. Miller, J.P. Krusic, D.A. Dixon, M.W. Reiff, H.J. Zhang, C.E. Anderson and J.A. Epstein, Radical ion salts of 2,3-dichloro-5,6-dicyanobenzoquinone and metallocenes. A reexamination of their magnetic and spectroscopic properties, J. Am. Chem. Soc,. 1986, 108, 4459-4466.

27 M. Hasani and M. Shamsipur, Interaction of 2,3-dichloro-5,6-dicyano-1,4-benzoquinone with aza-18-crown-6 and aza-12-crown-4 Kinetic and spectrophotometric studies in chloroform and acetonitrile solutions, J. Chem. Soc., Perkin Trans., 1998, 2, 1277-1281.

$28 \mathrm{M}$. Hasani and M. Shamsipur, Spectrophotometric study of interaction of 2,3-dichloro-5,6-dicyano-1,4-benzoquinone with diaza-18crown- 6 and diaza-15-crown-5 in acetonitrile and chloroform solutions, Spectrochim. Acta A, 2005, 61, 815-821.

29 M. E. Abdel-Hamid, M. Abdel-Salam, M. S. Mahrous and M. M. Abdel-Khalek, Utility of 2,3-dichloro-5,6-dicyano-p-benzoquinone in assay of codeine, emetine and pilocarpine, Talanta, 1985, 32, 1002-1004

30 K. M. Al-Ahmary, M. M. El-Kholy, I. A. Al-Solmy and M. M. Habeeb, Spectroscopic studies and molecular orbital calculations on the charge transfer reaction between DDQ and 2-aminopyridine, Spectrochim. Acta A, 2013, 110, 343-350.

31 R.S. Mulliken, Molecular compounds and their spectra. III. The interaction of electron donors and acceptors, J. Phys. Chem., 1952, 56, 801-822.
32 C. Reid and R.S. Mulliken, Molecular compounds and their spectra. IV. The pyridine-iodine system, J. Am. Chem. Soc., 1954, 76, 3869-3871.

33 S.N. Bhat, C.N.R. Rao, Kinetics of transformation of outer chargetransfer complexes to inner complexes, J. Am. Chem. Soc., 1966, 88, 3216-3219.

34 T. Nogami, K. Yoshihara, H. Hosoya and S. Nagakura, Chargetransfer interaction and chemical reaction. I. Reaction of aniline with chloranil, J. Phys. Chem., 1969, 73, 2670-2675.

35 R.S. Mulliken, Molecular compounds and their spectra. II, J. Am. Chem. Soc., 1952, 74, 811-824.

36 W.J. Lautenberger and J.G. Miller, Interaction of n-butylamine with tetracyanoethylene and chloranil, J. Phys. Chem., 1970, 74, 2722-2730.

37 T. Nagami, T. Yamaoka, K. Yoshihara and S. Nagakura, Charge transfer interaction and chemical reaction. III. Reactions of mPhenylenediamine and related compounds with chloranil, Bull. Chem. Soc. Jpn., 1971, 44, 380-386.

38 K. Kouno, C. Ogawa, Y. Shimomura and H. Yano, Y. Ueda, Interaction of imidazole derivatives with electron acceptors. II. Reaction products of imidazole with p-benzoquinone, Chem. Pharm. Bull., $1981,29,301-307$.

39 A.A. Frost and R.G. Pearson, Kinetics and Mechanism, 2nd edn., Wiley, New York, 1961.

40 J.L. Dye and V.A. Nicely, A general purpose curve fitting program for class and research use, J. Chem. Educ., 1971, 48, 443-448.

41 W.E. Wentworth, Rigorous least squares adjustment: application to some nonlinear equations, I and II, J. Chem. Educ., 1965, 42, 96-103, 162-167.

42 M.J.D. Powel, An efficient method for finding the minimum of a function of several variables without calculating derivatives, Comput. J., 1964, 7, 155-162.

43 S.H. Lin, K.P. Li and H. Eyring, in H. Eyring, D. Handerson, W. Yost (eds.), Physical Chemistry: An Advanced Treatise, vol. II, Academic Press, New York, 1977.

44 V. Gutmann, The Donor-Acceptor Concepts of Molecular Interactions, Plenum, New York, 1978

45 P. Job, Formation and stability of inorganic complexes in solution, Ann. Chim. Phys., 1928, 9, 113-203.

46 H.A. Benesi and J.H. Hildebrand, A spectrophotometric investigation of the interaction of iodine with aromatic hydrocarbons, J. Am. Chem. Soc., 1949, 71, 2703-2707.

47 A.M. Nour-el-Din, Charge-transfer complexes between beteroaromatic N-oxides and $\pi$-acceptors, Spectrochim. Acta A, 1985, 41, 1101-1104.

48 A.E. Mourad, $\pi-\pi$ Molecular complexes of $[2.2 .2](1,2,4)$ cyclophane and its methylated derivative with $\pi$-acceptors, Spectrochim. Acta A, 1983, 39, 289-292.

49 R.E. Merrifield and W.D. Philips, Cyanocarbon chemistry. II.1 Spectroscopic studies of the molecular complexes of tetracyanoethylene, J. Am. Chem. Soc., 1958, 80, 2778-2782.

$50 \mathrm{M}$. Hasani and S. Akbari, A spectrophotometric and thermodynamic study of the charge-transfer complexes of iodine with 2-aminomethyl-15-crown-5 in chloroform and 1,2-dichloroethane solutions, Spectrochim. Acta A, 2007, 67, 139-144.

$51 \mathrm{M}$. Hasani and A. Rezaei, Spectrophotometric study of the chargetransfer complexes of iodine with antipyrine in organic solvents, Spectrochim. Acta A, 2006, 65, 1093-1097.

$52 \mathrm{M}$. Hasani and S. Akbari, Spectrophotometric study of interaction of iodine with 4 -aminobenzo-15-crown-5 in chloroform, dichloromethane and 1,2-dichloroethane solutions, Spectrochim. Acta A, 2007, 68, 409-413.

53 G.G. Aloisi and S. Pignataro, Molecular complexes of substituted thiophens with $\sigma$ and $\pi$ acceptors. Charge transfer spectra and ionization potentials of the donors, J. Chem. Soc., Faraday Trans., 1973, 69, 534-539.

54 A.E. Mourad, Charge-transfer complexes of heterocyclic azines with П-acceptors, Spectrochim. Acta A, 1985, 41, 347-350.

55 R. Rathore, S.V. Lindeman and J.K. Kochi, Charge-transfer probes for molecular recognition via steric hindrance in donor-acceptor pairs, J. Am. Chem. Soc., 1997, 119, 9393-9404

56 I. Isenberg and S.L. Baird, Solvent effects in radical ion formation, J. Am. Chem. Soc., 1962, 84, 3803-3805. 